\title{
ANÁLISIS DENDROCRONOLÓGICO DE ÁRBOLES MUERTOS EN PIE DE LA ESPECIE PILGERODENDRON UVIFERUM FLORIN (CIPRÉS DE LAS GUAITECAS), EN EL SECTOR DE LAGO TORO, PROVINCIA DE ÚLTIMA ESPERANZA, REGIÓN DE MAGALLANES
}

\author{
DENDROCHRONOLOGICAL ANALYSIS OF STANDING DEAD TREES OF \\ PILGERODENDRON UVIFERUM FLORIN (CIPRÉS DE LAS GUAITECAS), IN LAKE \\ TORO AREA, ÚLTIMA ESPERANZA PROVINCE, MAGALLANES REGION \\ María Pía Guzmán Gaticaํㅜ, Pamela Soto Rogel² \& \\ Juan Carlos Aravena Donaire ${ }^{2,3}$
}

\begin{abstract}
This study reports the use of a dendrochronological approach to investigate the history of a stand of standing dead trees of Pilgerodendron uviferum (ciprés de las Guaitecas) in a locality of última Esperanza Province, Magallanes and Chilean Antarctica administrative Region. The analyzed trees were used to build of a floating chronology based on the concordant growth patterns of the samples, then this floating chronology was compared with a dated tree-ring chronology of the same species from Brunswick Peninsula in the same geographical region than the study area. The dendrochronological results allowed us to determine that the studied dead trees lived contemporaneously forming a multi-aged stand with continuous recruitment, suggesting that the trees were killed by repeated low-intensity forest fires occurred during the second half of the XIX century.
\end{abstract}

Key words: Pilgerodendron uviferum, tree-rings, forest fires.

\section{RESUMEN}

El presente estudio da cuenta del ejercicio de usar métodos dendrocronológicos para investigar la historia de un rodal de árboles muertos en pie de Pilgerodendron uviferum (ciprés de las Guaitecas) en una localidad de la provincia de Última Esperanza, región de Magallanes y Antártica Chilena. Los ejemplares analizados fueron utilizados para la construcción de una cronología flotante en base a los patrones de crecimiento concordantes de las muestras, luego esta cronología flotante fue comparada con otra

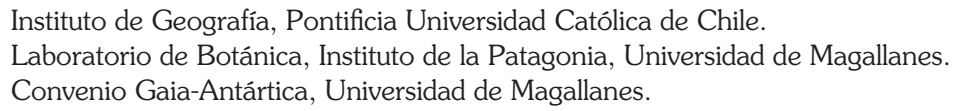


cronología de edad conocida de la misma especie desarrollada a partir de muestras colectadas en una localidad de la Península de Brunswick, en la misma región geográfica que el área de estudio. Los resultados dendrocronológicos nos permitieron determinar que los árboles estudiados son contemporáneos, que formaron un rodal multietáneo con reclutamiento continuo y sugieren que la muerte del rodal se habría producido por incendios forestales recurrentes de baja intensidad lo que determinó una mortalidad paulatina que abarcó varias décadas en la segunda mitad del siglo XIX.

Palabras clave: Pilgerodendron uviferum, anillos de crecimiento arbóreo, incendios forestales.

\section{INTRODUCCIÓN}

Pilgerodendron uviferum (D.Don) Florín es una especie monoespecífica, endémica del sur de Sudamérica, y en un estatus de conservación vulnerable de acuerdo a la UICN. En Chile se distribuye aproximadamente entre las latitudes de Valdivia $\left(40^{\circ} \mathrm{S}\right)$ y el Canal de Beagle $\left(55^{\circ} \mathrm{S}\right)$ cubriendo más de 1,600 km de distribución nortesur, siendo la conífera arbórea más austral del planeta. Abundante en la zona de los fiordos y canales patagónicos de la costa occidental del sur de Sudamérica, tolera condiciones de anegamiento de los suelos muy común en estas áreas geográficas. El estudio dendrocronológico de esta especie ha permitido estudiar su respuesta a las variaciones climáticas (Szeicz et al. 2000), sus patrones de crecimiento, y determinar edades máximas de 859 años para individuos vivos (Bannister et al. 2012). La especie es además muy resistente a la descomposición y en condiciones anóxicas puede preservarse por decenas de miles de años (Villagrán et al. 2004).

Todas las características mencionadas convierten a $P$. uviferum (nombre común ciprés de las Guaitecas) en un muy buen material para el estudio de las variaciones ambientales del pasado y para la reconstrucción de la historia y régimen de perturbaciones de los bosques de esta especie. Entre las perturbaciones más comunes están los incendios forestales, los que se reconocen como importantes agentes de perturbación y modeladores de la dinámica de los bosques del sur de Sudamérica (Veblen et al. 2011, Whitlock et al. 2007). Registros de incendios forestales se reconocen para todo el Holoceno -los últimos 10 mil años- a través de la acumulación de partículas de carbón en sedimentos de lagos y pantanos, y en heridas de fuego en troncos de árboles ( $\mathrm{Holz}$ et al. 2012; Moreno et al. 2014, Kitzberger \&
Veblen, 2003). En algunos casos estos registros preceden a la ocupación humana en el sur de Sudamérica y se asumen causas naturales en su origen, principalmente eventos climáticos como sequías y rayos, o erupciones volcánicas. Más hacia el presente, y ya con la presencia de poblaciones humanas de ocupación temprana de los ecosistemas australes, se asume una cada vez más importante influencia de las actividades asociadas a caza de fauna nativa en que los pueblos originarios usaron el fuego como método de apertura de la densa vegetación autóctona (Huber \& Markgraf, 2003). Las condiciones climáticas también determinaban la intensidad y distribución de los incendios resultantes. Moreno et al. (2014) ha definido ciclos centenarios en los que condiciones climáticas dominadas por fases tipo SAM positivo habrían facilitado la apertura de los bosques y una mayor frecuencia de incendios forestales. En los últimos siglos la alta frecuencia de incendios se asocia fuertemente a la transformación del paisaje impuesta por las actividades económicas de pobladores de origen europeo en la región austral. Para la provincia de Última Esperanza, en la que se ubica el área de estudio de este trabajo, la ocupación de origen europeo estuvo asociada a la instalación de pioneros primero, y luego a grandes estancias que utilizaron profusamente el fuego para despejar áreas para el forrajeo de ganado ovino (Martinic, 2002).

El objetivo general de este trabajo es construir una cronología flotante de ancho de anillos de crecimiento, a partir de muestras de rodelas de árboles muertos en pie de la especie Pilgerodendron uviferum colectadas en las cercanías del Lago Toro, en uno de los accesos al Parque Nacional Torres del Paine, e intentar estimar las edades absolutas de estos árboles conectando la referida cronología flotante, con cronologías de P. uviferum de edad conocida previamente desarrolladas para la región 


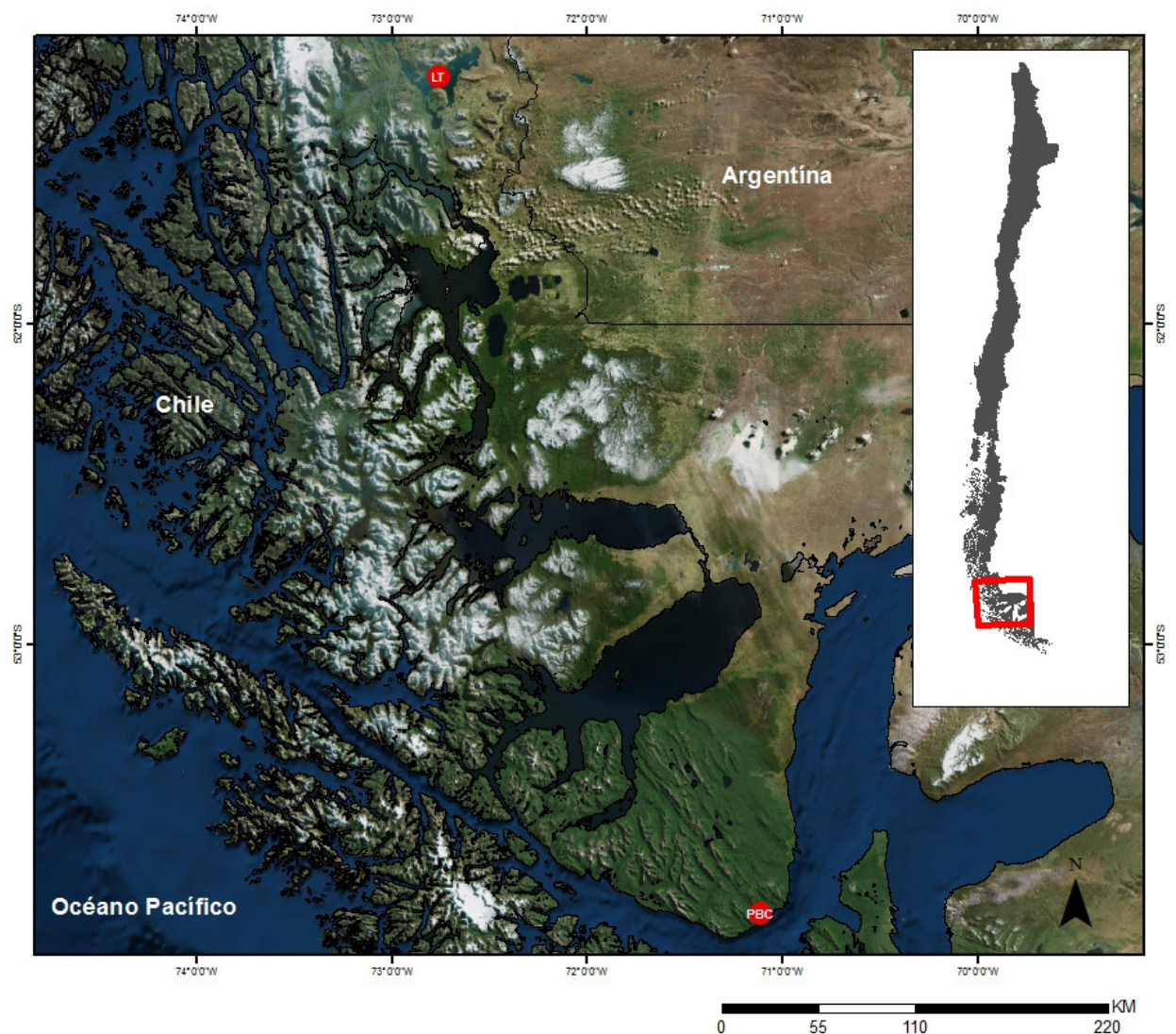

Fig. 1. Mapa del área de estudio. LT es la localidad Lago Toro donde se colectaron las muestras no fechadas objeto de este estudio y PBC es la localidad valle del Río San Nicolás donde se colectaron las muestras de la cronología de referencia de edad conocida (Aravena, 2007).

de Magallanes. De esta forma se pretende estimar si los árboles estudiados fueron contemporáneos, si su muerte obedece a una causa común y si es posible inferir aquella causa.

\section{MÉTODOS}

\section{Área de Estudio}

El estudio se realizó en una de las vías de acceso al Parque Nacional Torres del Paine, la ruta Y-290 corre por la costa oeste del Lago Toro, en el km 50 de dicha ruta (Fig. 1). Las coordenadas geográficas del área de estudio son $51^{\circ} 17$ 'S y $72^{\circ} 51^{\prime} \mathrm{O}$. La costa oeste del Lago Toro es una zona de alta diversidad vegetal con bosques abiertos, por efecto de incendios forestales recurrentes, dominados por Nothofagus betuloides (Mirb.) Oerst., Drimys winteri J. R. Forst. \& G. Forst.,
Maytenus magellanica Hook. f. y Embothrium coccineum J. R. Forst. \& G. Forst. Sin embargo, localmente se encuentran un alto número de especies no encontradas frecuentemente en la región tales como Lomatia ferruginea (Cav.) R. Br., Lomatia hirsuta (Lam.) Diels., Maytenus boaria Molina. En sitios de turberas de Sphagnum spp. y áreas aledañas a cuerpos de agua es común encontrar pequeños rodales de Pilgerodendron uviferum. Los incendios forestales pasados han afectado también a estos rodales de $P$. uviferum y es común encontrar en este sector agrupaciones de árboles de esta especie muertos en pie presumiblemente a causa de los incendios.

\section{Clima}

Por su ubicación al oriente de la Cordillera de los Andes el área de estudio se encuentra bajo 


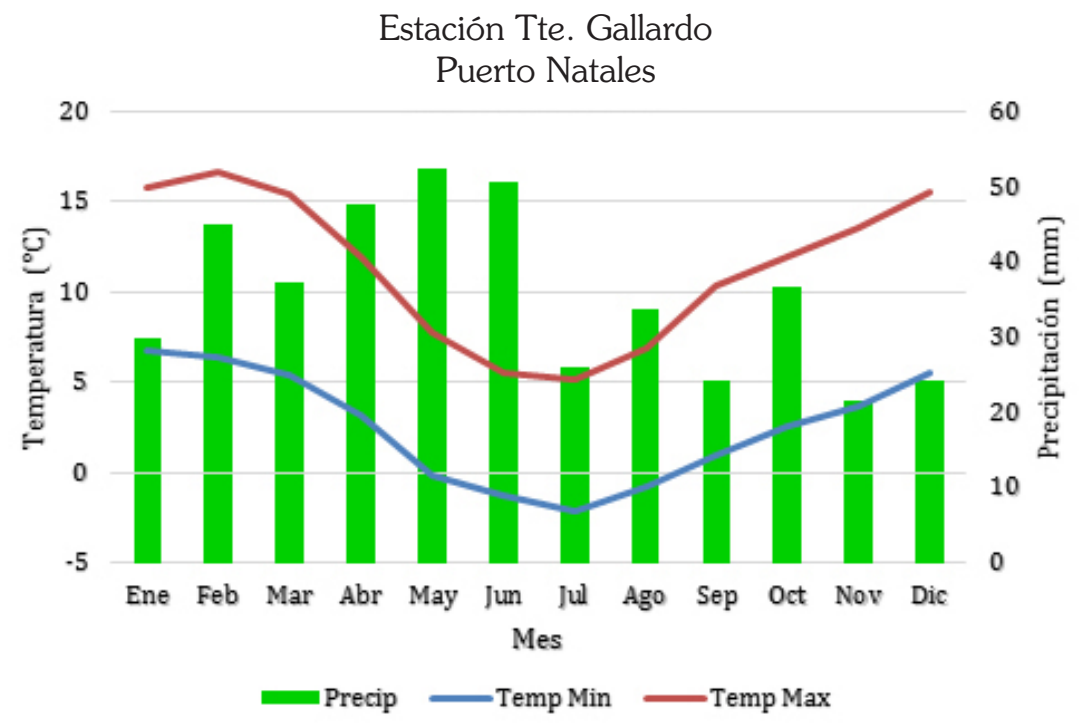

Fig. 2. Variaciones mensuales de precipitación (barras verdes), temperaturas del aire mínimas (línea azul) y máximas (línea roja) basadas en 11 años (2004-2014) de datos recopilados en la estación meteorológica del aeródromo Teniente Gallardo de Puerto Natales, provincia de Última Esperanza, Región de Magallanes y Antártica Chilena.

un clima trasandino con influencia esteparia. Esto determina niveles de precipitación mensual promedio máximos apenas superiores a $50 \mathrm{~mm}$ (Fig. 2), significativamente menores a los observados al oeste de la cadena montañosa andina, con montos mensuales de varios cientos de $\mathrm{mm}$, producto del efecto topográfico que esta cadena impone a los vientos prevalentes del oeste (Carrasco et al. 2002). Los datos climáticos provienen de la Estación Meteorológica del Aeródromo Teniente Gallardo de Puerto Natales, ubicada $50 \mathrm{~km}$ al sur del área de estudio.

El patrón mensual de variaciones de temperatura promedio del aire también refleja una condición de tipo continental con una marcada amplitud para valores invernales y estivales tanto de temperaturas máximas como mínimas (Fig. 2).

Colecta de las muestras

La colecta de muestras para el análisis de los patrones de crecimiento radial se realizó mediante una motosierra con una espada de 20 pulgadas de largo, lo que permitió la obtención de rodelas transversales de árboles muertos en pie de la especie P. uviferum. Todos los árboles se ubicaban en una superficie de alrededor de 2 hectáreas en una ladera de pendiente suave cercana al Lago
Toro (Fig. 1).

\section{Trabajo de laboratorio}

Las rodelas colectadas en terreno fueron transportadas al laboratorio para su procesamiento dendrocronológico. Este consistió en montar cada una de las rodelas en bases de madera mediante pegamento de modo de evitar que las rodelas se fracturen. Luego que las muestran se fijan a las bases de madera se procede a lijar la superficie transversal que queda expuesta con lijas de grano cada vez más fino, con lo que se consigue que las rodelas queden pulidas de modo de obtener una visibilidad óptima de la superficie a examinar (Fig. 3).

Bajo lupa estereoscópica con un aumento de hasta 40X se marcaron y contaron los anillos de crecimiento de cada una de las rodelas. Para esto se marcaron las decenas (un punto), cincuentenas (dos puntos), y centenas (tres puntos) de anillos para un mejor control del marcado. Luego se trazaron a lo menos dos radios en cada una de las rodelas escogiendo aquellas porciones con mejor visibilidad. Usando los radios escogidos se procedió a medir el ancho de cada anillo de crecimiento mediante una tabla de medición marca Velmex en el Laboratorio 


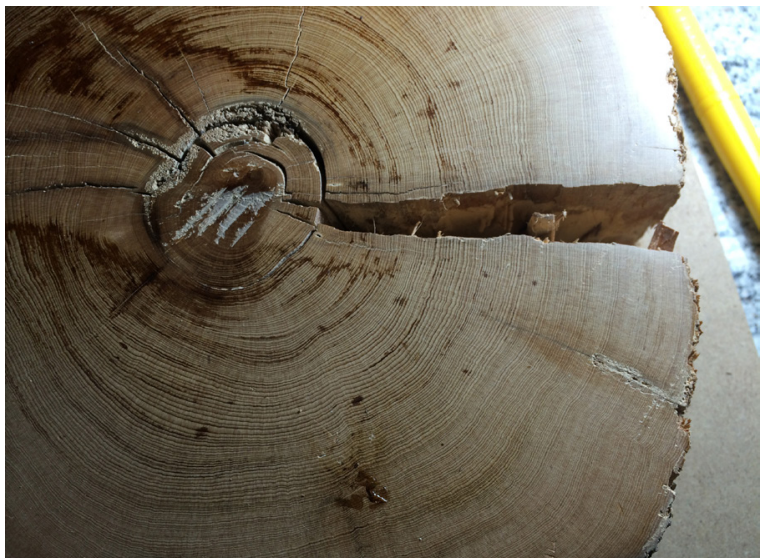

Fig. 3. Muestra de las rodela de Ciprés de las Guaitecas analizada en laboratorio, donde se pueden apreciar los anillos de crecimiento.

de Dendrocronología de la Fundación CEQUA, utilizando el softward Medition 12X que entrega los valores medidos en formato digital. De esta forma se obtuvieron al menos dos series de ancho de anillos de crecimiento por cada rodela.

Construcción de cronología flotante.

Las series de medición de ancho de anillos de crecimiento obtenidas fueron analizadas usando el programa computacional COFECHA (Holmes, 1982). El programa permite mediante análisis estadísticos comparativos entre las muestras medidas hacer coincidir sus patrones de crecimiento, los que se espera muestren un patrón común si son contemporáneos, e identificar los errores de medición si es que existiesen, generalmente referidos a anillos faltantes o sobrantes en las muestras. Este proceso de identificación de un patrón de crecimiento común entre las series de crecimiento de distintos árboles, permite asignar los anillos de crecimiento a estaciones de crecimiento contemporáneas, y se denomina "cofechado". La identificación de este patrón común además permite estimar el crecimiento promedio para cada uno los anillos de crecimiento, obteniendo así una serie o cronología promedio para el período analizado. Como en el caso de las rodelas estudiadas en este trabajo no se conoce las edades calendario de sus anillos, la coincidencia relativa entre sus series no está "anclada" a una fecha determinada, y por lo tanto a la serie promedio resultante se le denomina "cronología flotante".

\section{Fechado absoluto de cronología flotante}

Para poder asignar edades absolutas a las muestras estudiadas, se debió comparar usando el programa COFECHA la cronología flotante obtenida a partir de las rodelas con una cronología de edades conocidas de árboles de la misma especie obtenida previamente para la región de Magallanes. Como cronología fechada de referencia se escogió la cronología PBC obtenida a partir de muestras de $P$. uviferum colectadas en el valle del Río San Nicolás, Península de Brunswick (Aravena, 2007). La referida cronología fue escogida por la extensión temporal de la misma la que asegura una mayor probabilidad de sobreposición temporal con la cronología flotante y por encontrarse en condiciones ecológicas similares (Fig. 1).

\section{RESULTADOS}

El análisis de las rodelas incluyó 12 árboles muertos en pie, con un total de 24 radios medidos. La Tabla 1 muestra la identificación de los radios medidos con el número de anillos en cada caso y la observación si se trata de radios completos, alcanzando el último anillo formado antes de la muerte del árbol, o si producto de la erosión o falta de nitidez por compresión de la porción externa del crecimiento el radio medido es incompleto.

De la Tabla 1 se verifica que en 10 de los 12 árboles analizados fue posible conseguir edades completas, a partir de alguno de sus radios. Esto nos permite tener una idea de la estructura de edades de los árboles analizados como se muestra en la Fig. 4.

Las frecuencias de edades se distribuyen en el rango 227 a 369 años con una amplia dispersión de las edades, evidenciando un bosque multietáneo con reclutamiento continuo de individuos asociado a regímenes de perturbación de pequeña escala. La inferencia está hecha sin embargo con base en un número bajo de individuos muestreados.

La comparación de los patrones de crecimiento de las rodelas de árboles muertos permitió cofechar las series medidas y comprobar que éstas eran contemporáneas con sobreposición de sus períodos de crecimiento para la casi totalidad de los casos. En la Tabla 1 se indica las correlaciones de cada una de las series medidas 
Tabla 1. Identificación de las muestras analizadas, con el número de años medidos en cada caso y con la indicación si se trata de radios completos o incompletos. Se indica además la correlación de cada muestra con la serie promedio.

\begin{tabular}{cccc}
\hline Nombre Muestra & Número de anillos & Condición del radio & Correlación con Serie promedio \\
\hline LT_01RA & 227 & Completo & 0.546 \\
LT_01RB & 105 & Incompleto & 0.499 \\
LT_02RA & 234 & Incompleto & 0.463 \\
LT_02RB & 191 & Incompleto & 0.560 \\
LT_03RA & 305 & Incompleto & 0.458 \\
LT_03RB & 327 & Completo & 0.456 \\
LT_06RA & 369 & Completo & 0.473 \\
LT_09RA & 275 & Incompleto & 0.601 \\
LT_09RB & 333 & Completo & 0.586 \\
LT_10RA & 334 & Completo & 0.496 \\
LT_10RB & 344 & Completo & 0.522 \\
LT_13RA & 242 & Completo & 0.604 \\
LT_13RB & 248 & Completo & 0.607 \\
LT_15RA & 257 & Completo & 0.384 \\
LT_15RB & 290 & Completo & 0.450 \\
LT_16RB & 273 & Incompleto & 0.376 \\
LT_17aRA & 240 & Completo & 0.596 \\
LT_17aRB & 213 & Incompleto & 0.536 \\
LT_17bRA & 306 & Completo & 0.384 \\
LT_17bRB & 255 & Incompleto & 0.542 \\
LT_17bRC & 206 & Incompleto & 0.533 \\
LT_17bRD & 313 & Incompleto & 0.448 \\
LT_19RA & 216 & Incompleto & 0.619 \\
LT_19RB & 244 & 0.560 \\
\hline
\end{tabular}

con la serie promedio. Nótese que el valor de correlación límite para un nivel de confianza de un $99 \%$ es de 0.3281 . Los valores de correlación para las series medidas están en el rango de 0.376 a 0.619 con un promedio de 0.484 , todos altamente significativos. Este buen nivel de correlación entre las series comparadas nos permite generar una serie de referencia o promedio, o cronología "flotante" dado que sus anillos no están asociados a fechas absolutas (Fig. 5).

Para conseguir el objetivo específico de obtener las edades absolutas de las muestras analizadas se comparó los patrones de crecimiento de estas muestras con el patrón de crecimiento de una cronología longeva de $P$. uviferum desarrollada a partir de muestras de árboles vivos y por lo mismo de edades calendario conocidas. Para ello se escogió la cronología desarrollada para los bosques de ciprés de las Guaitecas de la localidad Río San Nicolás, en la Península de Brunswick, región de Magallanes (a la que se le asignó el código PBC; Aravena, 2007), cuyas coordenadas geográficas de referencia son $53^{\circ} 49^{\prime} \mathrm{S}$ y $72^{\circ} 07^{\prime} \mathrm{O}$, alrededor de $300 \mathrm{~km}$ al sur del área de estudio de Lago Toro. Dicha cronología cubre el período de 486 años desde el año 1517 al 2002. Para el desarrollo de esta cronología se utilizaron 28 muestras y su intercorrelación fue de 0.696 (Aravena, 2007). La Fig. 6 muestra la cronología de P. uviferum para la localidad de Río San Nicolás, mostrando las 


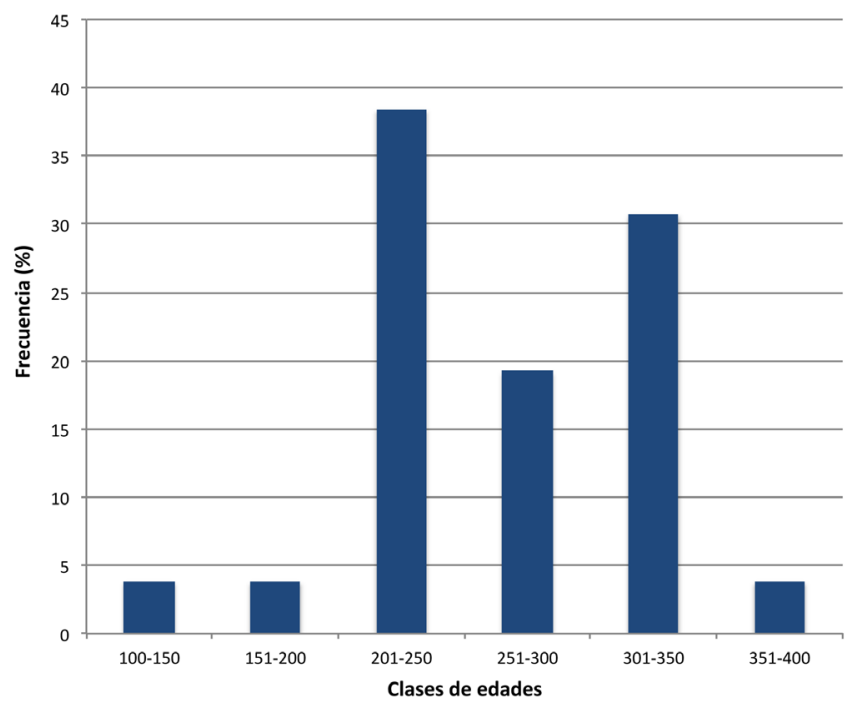

Fig. 4. Frecuencia de edades completas en clases de 20 años, expresado en porcentaje del total de árboles analizados ( $\mathrm{N}=10)$.

principales variaciones en el crecimiento de estos árboles.

Esta cronología de referencia del valle del Río San Nicolás es representativa de los patrones de crecimiento de toda la región según lo indican los análisis de correlación con la red de cronologías de $P$. uviferum actualmente desarrollada (Aravena, 2007). El examen de la Fig. 6 permite observar la recurrencia de valores de marcada disminución en el crecimiento con mínimos para los años 1558, 1607, 1877 y 1978. También llama la atención el período 1644-1654 como el más prolongado de crecimiento suprimido de toda la serie. Estos años son indicadores de la respuesta del crecimiento de esta especie a eventos ambientales extremos en algunos casos de alcance regional.

El mismo programa COFECHA antes descrito permitió comparar los patrones de crecimiento de la cronología fechada con las muestras que formaron la cronología flotante. El análisis de correlación entre ambas cronologías comprobó que el mejor ajuste se logra con una

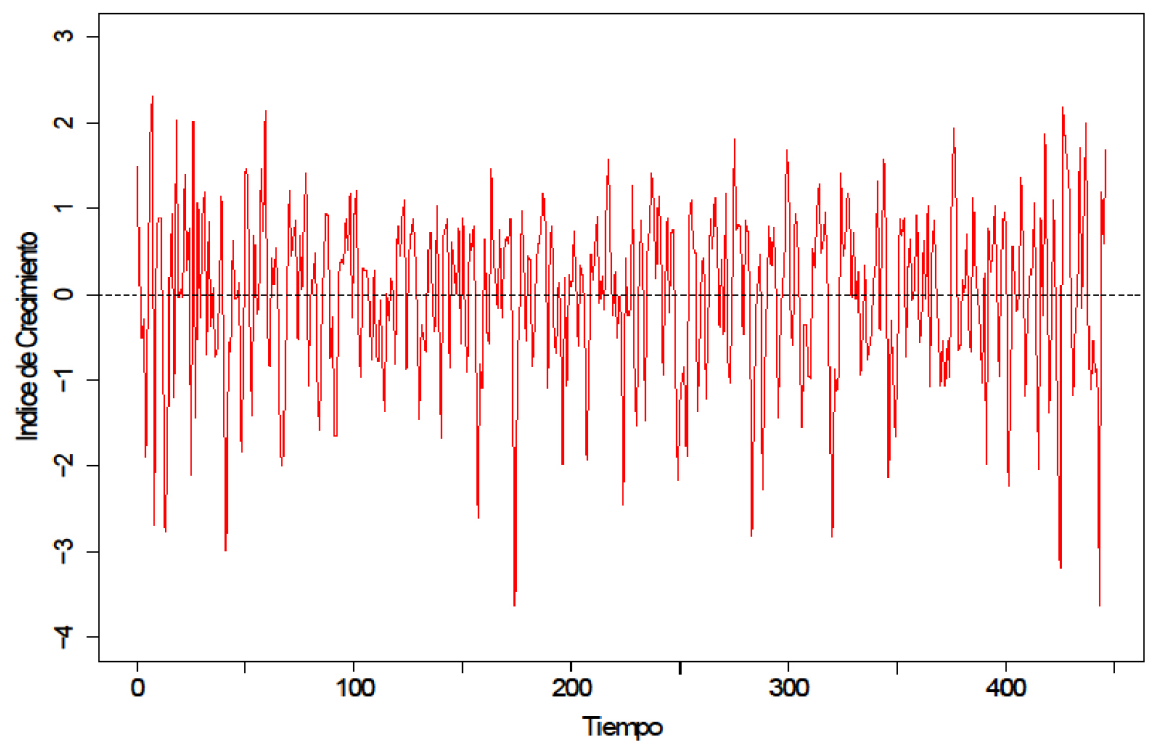

Fig. 5. Cronología flotante obtenida a partir del promedio de crecimiento por año de las rodelas no fechadas de P. uviferum. 


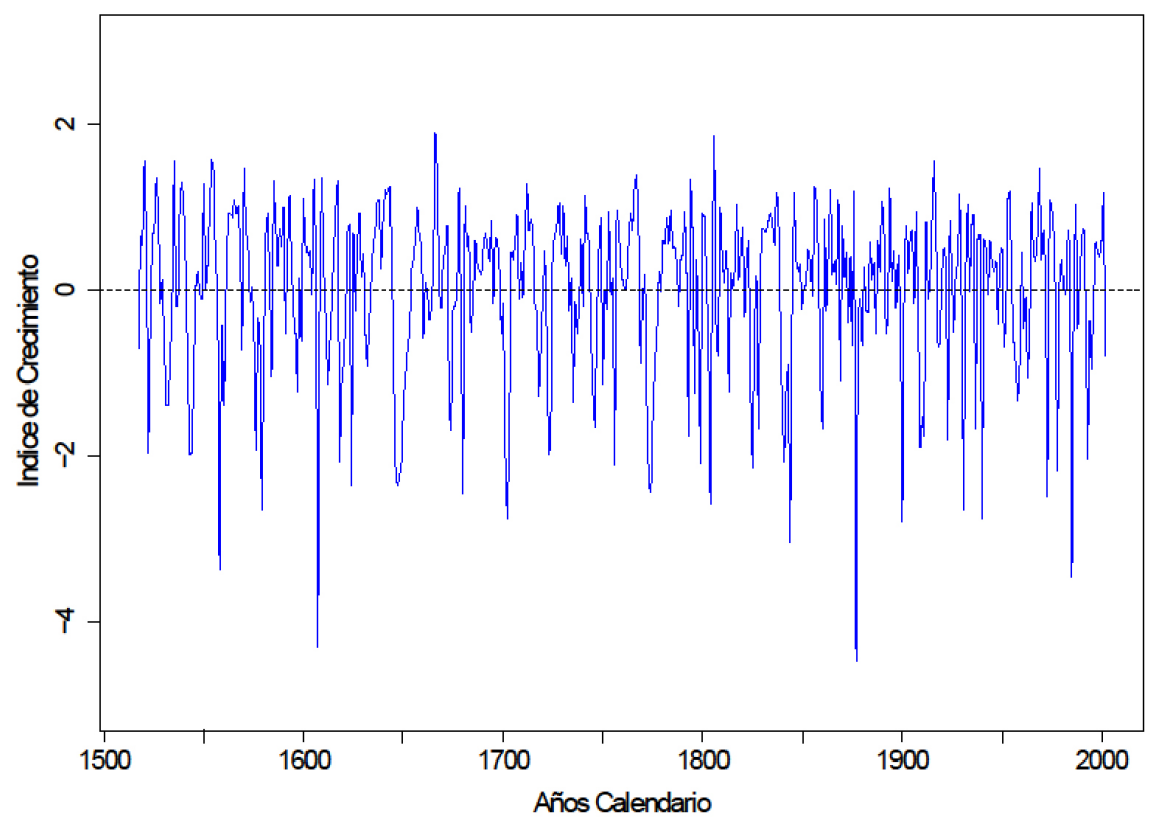

Fig. 6. Cronología de ancho de anillos de P. uviferum de la localidad de Río San Nicolás (PBC), Península de Brunswick, Región de Magallanes.

superposición de 380 años de 1517 a 1896 (Fig. además la información de las fechas absolutas 7). Esta sobreposición nos permitió "anclar" la para cada una de las muestras de la cronología de cronología flotante asignándole fechas calendario Lago Toro (Fig. 8). La mayoría de las muestras entre 1459 y 1896. parten alrededor o después del año 1550 y sólo

El anclaje de las cronologías nos entrega tres árboles tienen edades de reclutamiento

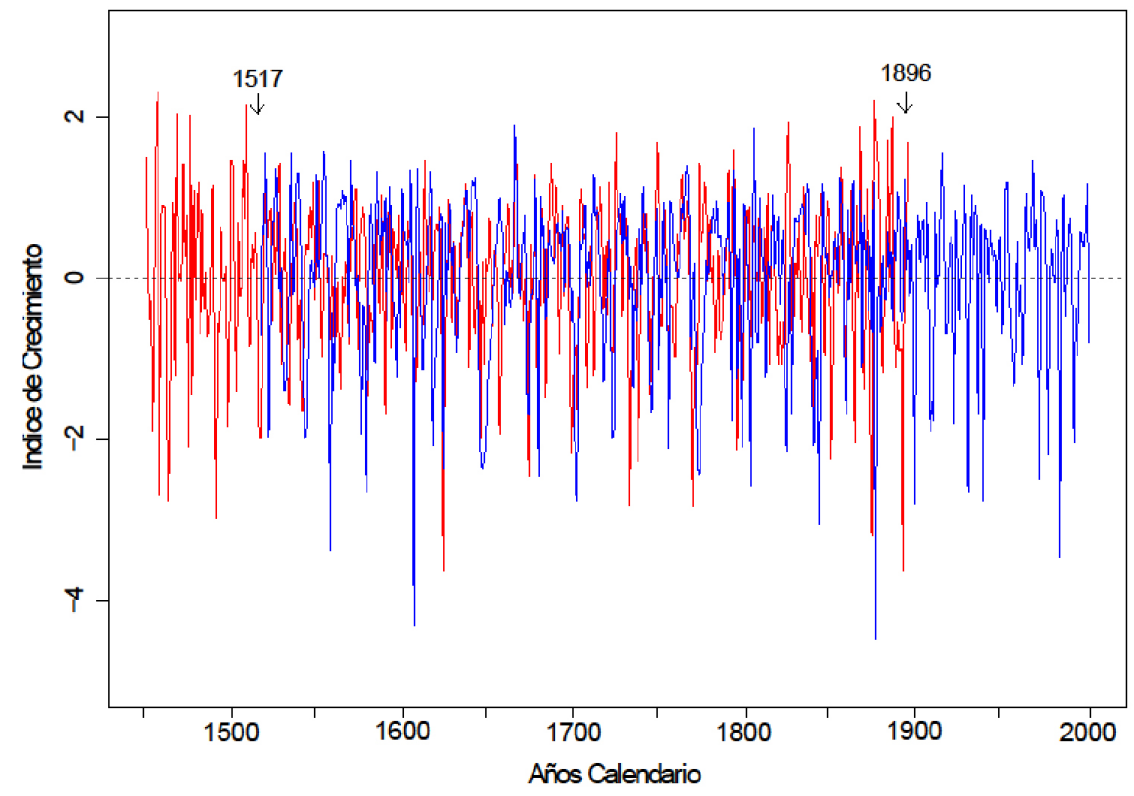

Fig. 7. Superposición de cronología de Lago Toro (línea roja; 1459-1896) con la cronología fechada de Río San Nicolás (línea azul; 1517-2002). Se indican los años de inicio y término de la superposición entre ambas cronologías. 


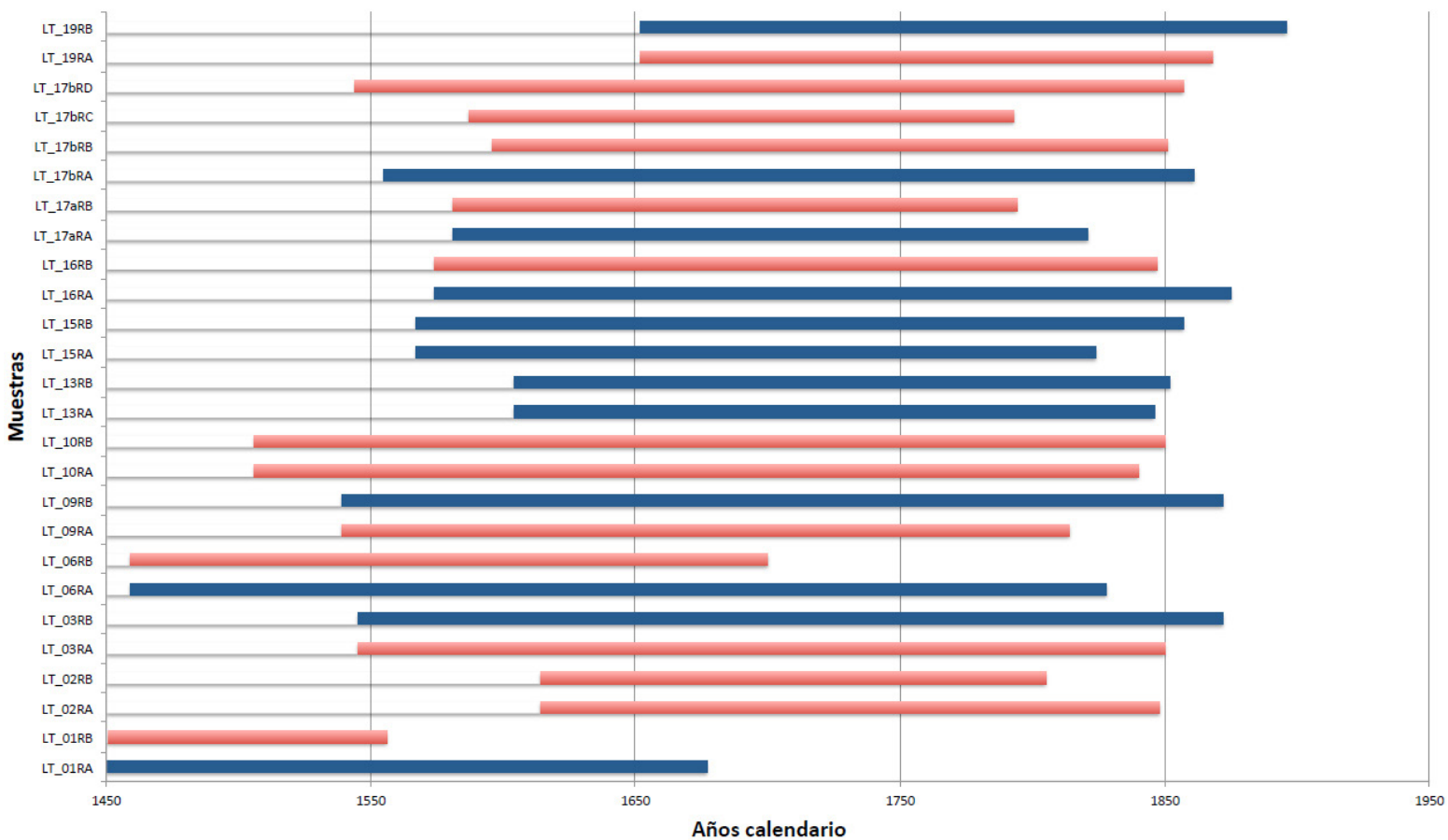

Fig. 8. Muestras de la cronología Lago Toro indicando sus fechas absolutas. En azul se muestran las series completas, mientras que en color rojo las series incompletas por pérdida de nitidez hacia la periferia de la muestra o por erosión de la misma.

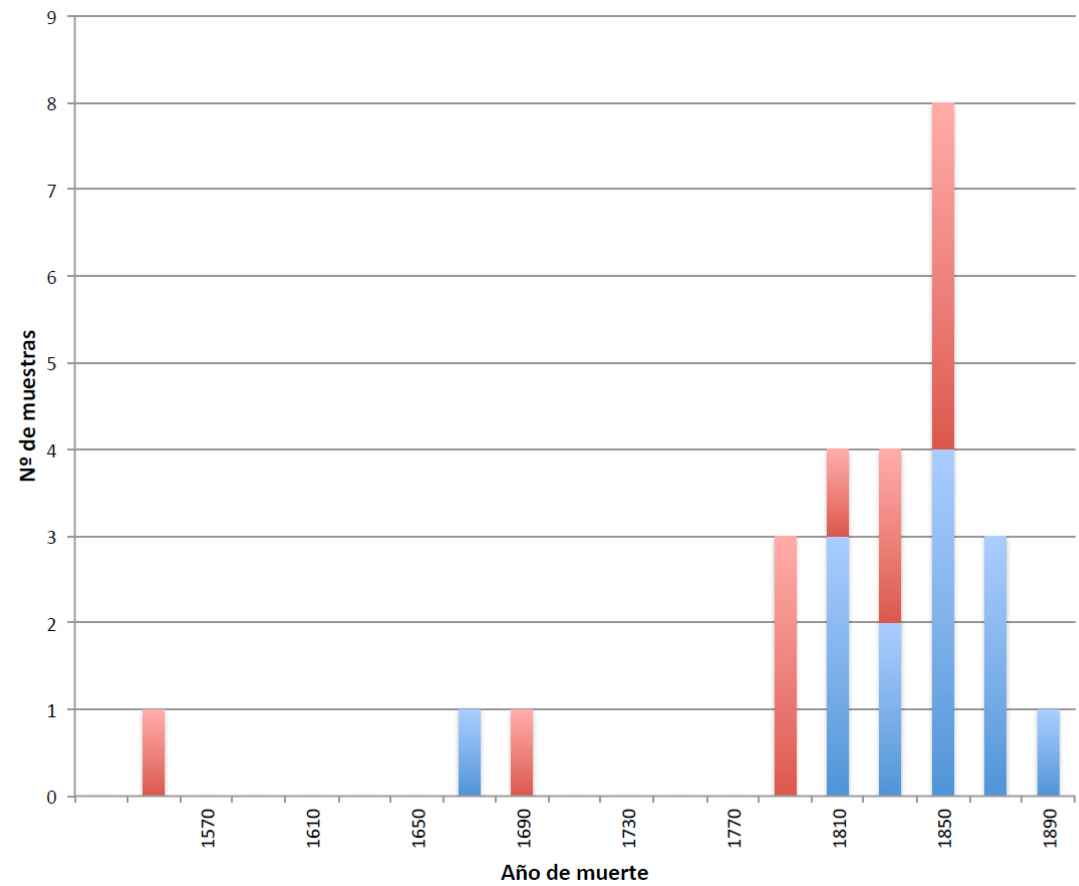

Fig. 9. Año de muerte de los árboles expresado en clases de edad de 20 años. En azul muestras completas y en rojo muestras incompletas. 
anteriores. Tanto en la Fig. 8 como en la Figura 9 además es posible observar las fechas de muerte de las muestras completas. La mayor frecuencia de muerte de árboles (69\%) según estos antecedentes murió entre los años 1820 y 1879. Esto representa un rango amplio de fechas de muerte e indica una mortalidad no instantánea sino más bien paulatina.

De esta manera se confirma el resultado que aunque el bosque analizado estuvo formado por individuos que coexistieron la mayor parte de su ciclo vital, las muertes de los árboles estudiados no habría ocurrido producto de un evento único.

\section{DISCUSIÓN}

El área estudiada en este trabajo presenta una vegetación de transición entre los bosques húmedos dominados por Nothofagus betuloides y matorral caducifolio de Nothofagus antárctica (ñirre), y se encuentra próximo a ambientes de estepa seca (ver Fig. 1). La proximidad a la estepa hace que esta zona esté además sujeta a incursiones de tormentas convectivas del oriente con un aumento asociado de rayos (Garreaud et al. 2014). Todos estos factores sumados a condiciones de sequía $\mathrm{y} / \mathrm{o}$ aumento de las temperaturas, y uso del fuego por poblaciones humanas, configurarían condiciones propicias para la ocurrencia de incendios forestales. Moreno et al. (2014) a partir de un registro de sedimentos de fondo de lago en la misma localidad de este trabajo describen ciclos centenarios de alternancia de fases húmedas y frías, con períodos cálidos y secos. Esta alternancia climática estaría modulada por patrones de circulación atmosférica del tipo "Modo Anular del Sur" (SAM según su sigla en inglés). La fase de predominio tipo SAM positivo para los últimos cientos de años estarían determinando condiciones climáticas con predominio cálido y seco.

Observaciones de terreno durante la colecta de las muestras analizadas en este trabajo arrojaron abundante evidencia de incendios recurrentes en nuestra área de estudio (Fig. 10). Por lo tanto una primera aproximación al problema de la determinación de la causa de muerte de los árboles estudiados sugiere que ésta no fue producto de un evento catastrófico único, si no que fue

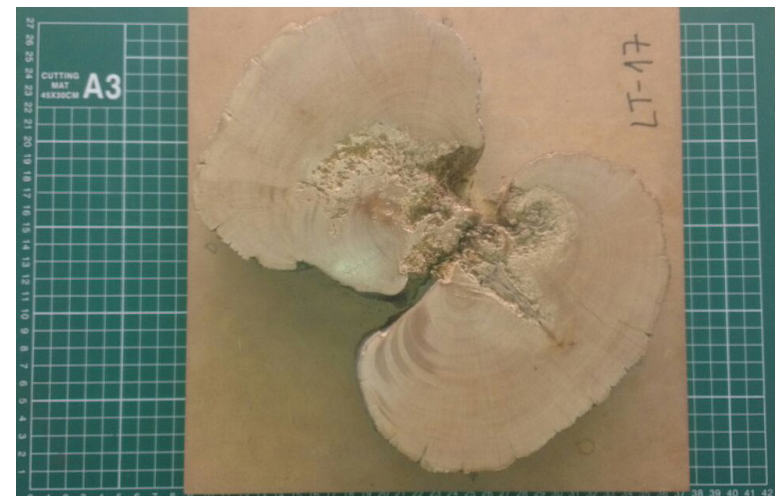

Fig. 10. Muestra de una rodela de ciprés de las Guaitecas analizada en laboratorio, donde se pueden apreciar las cicatrices dejadas por los incendios (flechas negras).

ocurriendo de forma paulatina, posiblemente una sucesión de incendios recurrentes de baja intensidad. Estos incendios recurrentes pudieran haberse debido a condiciones climáticas especiales como años extremos cálidos y secos unidos a tormentas eléctricas convectivas productoras de rayos (Garreaud et al. 2014). Tampoco puede descartarse fuegos producidos tanto por los pueblos originarios que cazaban guanacos con fuego, o por los primeros baqueanos chilenos que transitaban por el sector a partir de los 1870s antes de la instalación de las grandes estancias ovejeras de la provincia de Última Esperanza pasado el año 1890 (Martinic, 2002; Huber \& Markgraf, 2003). El poblamiento colonizador de origen europeo llegó al distrito de Última Esperanza en 1894 donde formaron un importante establecimiento de crianza de ovejas en los márgenes del lago Toro, y utilizaron intensivamente el fuego para la apertura de campos (Martinic, 1984).

Por último, la capacidad de Pilgerodendron uviferum de crecer en ambientes muy humedos y ser la especie arbórea dominante en sitios anegados refuerza la posibilidad de que este tipo de bosquetes haya resistido varios incendios de baja intensidad que fueron provocando la muerte paulatina de los árboles analizados.

\section{CONCLUSIONES}

De los resultados obtenidos en este trabajo concluimos que:

- La mayoría de los árboles analizados en 
este estudio vivieron $y$ crecieron contemporáneamente entre los siglos XVI a XIX, en los alrededores del Lago Toro, conformando un bosque adulto de Pilgerodendron uviferum de dominancia local, en un ambiente anegado y rodeado de bosque siempreverde dominado por Nothofagus betuloides.

- El área de estudio estuvo sujeta a incendios recurrentes y de poca intensidad. Estos incendios forestales pudieron haber sido causados por condiciones climáticas especiales, y/o la acción humana tanto de pueblos originarios como de pioneros de origen europeo.

- Por último, se presume que debido a que las muertes de los árboles analizados ocurrieron esporádicamente, la causa de estos fallecimientos ocasionales fue probablemente una sucesión de eventos catastróficos, presumiblemente incendios recurrentes.

\section{AGRADECIMIENTOS}

Agradecemos al proyecto Fondecyt 1130381 por financiar parcialmente la estadía de María Pía Guzmán en el laboratorio de Botánica del Instituto de la Patagonia de la Universidad de Magallanes, a la Fundación CEQUA por permitir el uso de sus dependencias y equipos de medición dendrocronológica, y a Mauricio Fuentes P. por su ayuda en la colecta de muestras en el sitio de estudio. Esta es una contribución al Programa de Mejoramiento Institucional GAIA-Antártica de la Universidad de Magallanes.

\section{LITERATURA CITADA}

Aravena, J.C. (2007). Reconstructing climate variability using tree rings and glacier fluctuations in the Chilean Andes. Ph.D thesis submitted in partial fulfillment of the requirements for the degree of Doctor of Philisophy.

Bannister, J.R., Donoso P., \& Bauhus, J. (2012). Persistence of the slow growing conifer Pilgerodendreon uviferum in old-growth and fire-disturbed southern bog forests. Ecosystems 15: 1158-1172.

Carrasco, J., Casassa, G., \& Rivera, A. (2002). Meteorological and climatological aspects of the Southern Patagonia Ice Cap, América del Sur. En K. Academic/Plenum, G. Casassa, F. Sepulveda, \& M. Sincler (Edits.), The Patagonian Icefields: A unique natural laboratory for environmental and climate change studies (págs. 29-41).

Garreaud, R., M.G. Nicora, R.E. Burgesser, E.E. Avila (2014). Lightning in Western Patagonia. Journal of Geophysical Research. Atmospheres.

Holmes R.L. (1982). Computer-assisted quality control in tree-ring dating and measurement. Tree-Ring Bulletin 44: 69-75.

Holz, A., Haberle, S., Veblen, T. T., De PolHolz, R., \& Southon, J. (2011). Fire history in western Patagonia from paired tree-ring fire-scar. Climate of the Past, 8(2): 451-466.

Huber, U.M., \& Markgraf, V. (2003) European impact on fire regimes and vegetation dynamics at the steppe-forest ecotone of southern Patagonia. The Holocene 13(4): 567-579.

Kitzberger, T., \& Veblen, T. (2003). Influences of climate on fire in northern Patagonia, Argentina. En T. Veblen, W. Baker, G. Montenegro, \& T. Swetnam, Fire regimes and climatic change in temperate ecosystems of the Western Americas (págs. 290-315). Springer-Verlag.

Martinic, M. (1984). El hombre y el uso del medio natural en Magallanes: Una visión histórica. Revista Ambiente y Desarrollo, 1(1), 6173.

Martinic, M. (2002). La participación de capitales británicos en el desarrollo económico del territorio de Magallanes (1880-1920). Historio, 35, 299-321.

Moreno, P., Vilanova, I., Villa-Martínez, R., Garreud, R., Rojas, M., \& De Pol-Holz, R. (2014). Southern Annular Mode-like changes in southwestern Patagonia at centennial timescales over the last three millennia. Nature Communications, DOI: 10.1038/ncomms5375.

Szeicz, J., Lara A., Díaz, S., \& Aravena, J.C. (2000). Dendrochronological studies of Pilgerodendron uviferum in southwestern South America. In Dendrocronología en 
América Latina. F.A. Roig (Compilador); Villagrán, C., León, A., \& Roig, F. (2004). PalEDIUNC Mendoza, Argentina: 245-270.

Veblen, T., Holz, A., Paritsis, J., Raffaele, E., Kitzberger, T., \& Blackhall, M. (2011). Adapting to global environmental change in Patagonia: What role for disturbance ecology? Austral Ecology, 36, 891-903. eodistribución del alerce y ciprés de las Guaitecas durante períodos interestadiales de la Glaciación Llanquihue: provincias de Llanquihue y Chiloé, Región de Los Lagos, Chile. Revista Geológica de Chile, 31(1), 133-151. 